\title{
Comparison of the Rayleigh and K-Distributions for Application in Incoherent Change Detection
}

\author{
Natanael Rodrigues Gomes $\left.{ }^{(}\right)$, Patrik Dammert, Senior Member, IEEE, Mats Ingemar Pettersson ${ }^{\circledR}$, Member, IEEE, \\ Viet Thuy $\mathrm{Vu}^{\circledR}$, Member, IEEE, and Hans Hellsten
}

\begin{abstract}
The aim of this letter is to compare two incoherent change-detection algorithms for target detection in low-frequency ultrawideband (UWB) synthetic aperture radar (SAR) images. The considered UWB SAR operates in the frequency range from 20 to $90 \mathrm{MHz}$. Both approaches employ a likelihood ratio test according to the Neyman-Pearson criterion. First, the bivariate Rayleigh probability distribution is used to implement the likelihood ratio test function. This distribution is well known and has been used for change-detection algorithms in low-frequency UWB SAR with good results. Aiming to minimize the false alarm rate and taking into consideration that low-frequency UWB SAR images have high resolution compared to the transmitted wavelength, the second approach implements the test by using a bivariate K-distribution. This distribution has scale and shape parameters that can be used to adjust it to the data. No filter is applied to the data set images, and the results show that with a good statistical model, it is not needed to rely on filtering the data to decrease the number of false alarms. Therefore, we can have a better tradeoff between resolution and detection performance.
\end{abstract}

Index Terms-Change detection, likelihood ratio test, synthetic aperture radar (SAR).

\section{INTRODUCTION}

$\mathbf{T}$ HE extraction and analysis of information from radar images have several important applications. For example, one can consider the surveillance of large forest areas, where targets can be concealed by foliage and the tree canopy [1], [2]. In this area, change-detection methodology, which concerns the problem of detecting targets, has been well established by many publications (see [3]-[6]). The methodology remains a subject of interest to many experts because of different applications of target detection. A summary of change-detection methodology is given in [7]-[10]. Generally, the solutions work well, but the problem is not observed from the resolution cell perspective. Therefore, few works have implemented and compared multivariate probability density functions (PDFs)

Manuscript received July 16, 2018; revised November 5, 2018; accepted November 8, 2018. Date of publication December 5, 2018; date of current version April 22, 2019. This work was supported in part by the Brazilian Agencies CISB and CNPq, in part by Saab Electronic Defence Systems, in part by Swedish Defence Research Agency, and in part by KK-Foundation. (Corresponding author: Natanael Rodrigues Gomes.)

N. R. Gomes is with the Department of Electronics and Computer, Federal University of Santa Maria, Santa Maria 97105-900, Brazil (e-mail: natanael-rodrigues.gomes@ufsm.br).

P. Dammert and H. Hellsten are with Saab AB (Surveillance), 41289 Gothenburg, Sweden.

M. I. Pettersson and V. T. Vu are with the Department of Mathematics, Blekinge Institute of Technology, 37179 Karlskrona, Sweden.

Color versions of one or more of the figures in this letter are available online at http://ieeexplore.ieee.org.

Digital Object Identifier 10.1109/LGRS.2018.2881733 to take into account the clutter correlation in high-resolution systems and, in particular, in low-frequency ultrawideband (UWB) synthetic aperture radar (SAR), where the scatterers are stable between illuminations and the radar resolution cell contains only a few scatterers. The decision rule in several change-detection algorithms is a statistical hypothesis test. The decision of whether a change has occurred at a given pixel corresponds to choosing between two competing hypotheses, i.e., the null hypothesis and the alternative hypothesis, which correspond to no change and change decisions, respectively. Knowing the conditional joint PDF enables the selection of the hypothesis that best describes the intensity change via hypothesis testing [3], [4], [11].

In this letter, the main goal is to compare two different statistical models that can be used in a likelihood ratio test for change detection. One model uses the bivariate Rayleigh distribution to model the clutter on the reference and surveillance SAR images; the other employs the bivariate K-distribution. In general, the bivariate Rayleigh distribution is based on the assumption that the scattered return from clutter comes from a large number of scatterers [3]. Therefore, according to the central limit theorem, the appropriate statistical model for clutter approximates a Gaussian model, and its amplitude is well represented by the Rayleigh distribution. However, taking into account the cell resolution, the clutter statistics for many modern SAR systems cannot be considered to be Gaussian distributed. Instead, due to random fluctuations in the number of scatterers, the clutter distribution can be approximated by the K-distribution, and it is very important to consider this fact in a UWB SAR system, where the high resolution in the azimuth and range can be achieved simultaneously. In this case, a lower false alarm rate (FAR) is expected from the detection algorithm due to the heavy-tailed characteristic of the K-distribution.

To present and compare the results from applying the two distributions, this letter is organized as follows. Initially, Section II presents a succinct statistical analysis related to the distribution of SAR image amplitudes. Section III is dedicated to the mathematical formulations used to implement change detection. In Section IV, the likelihood function for the bivariate Rayleigh distribution is presented. The bivariate K-distribution from spherically invariant random processes (SIRPs) is described in Section V. The tests and experimental results are presented and discussed in Section VI. Finally, concluding remarks are given in Section VII. 


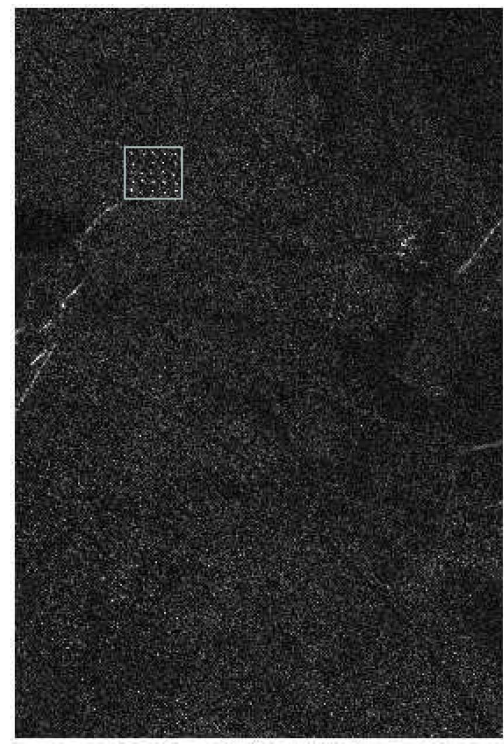

Fig. 1. SAR image from the data collected by CARABAS [5]. A rectangle is used to point out the target regions in the image.

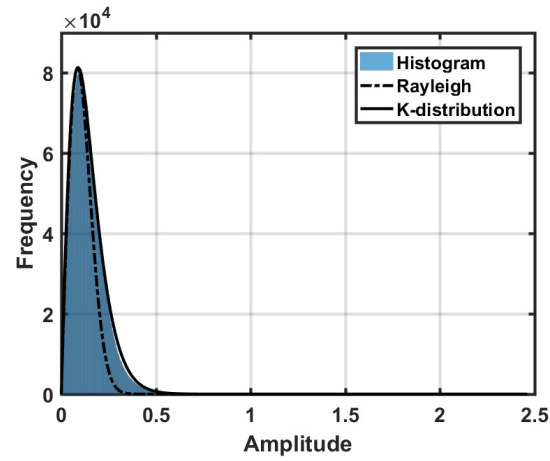

Fig. 2. Histogram of the SAR image and PDF of the Rayleigh distribution and K-distribution.

\section{SAR IMAGE STATISTICS}

A histogram can provide information about the shape of the distribution of SAR image amplitudes. The histogram of the image in Fig. 1 is presented in Fig. 2. As observed in Fig. 2, the distribution of SAR image amplitudes is close to the PDF of the Rayleigh and K-distribution. This characteristic indicates that these two distributions could be suitable models of the amplitude distribution of SAR images. However, note that the histogram is made over the whole SAR image; therefore, different areas with entirely different clutter statistics are added together in the histogram. The K-distribution can handle these global statistics better than can the Rayleigh distribution, as shown in Fig. 2. In this letter, however, the statistical tests are conducted locally over a local image.

The PDF for the Rayleigh distribution, as exemplified in Fig. 2, is expressed by the following equation:

$$
f(h ; \sigma)=\frac{h}{\sigma^{2}} \exp \left(-\frac{h^{2}}{2 \sigma^{2}}\right)
$$

where $h$ denotes the image amplitude, and $\sigma>0$ is the scale parameter of the PDF. There are several methods to estimate the scale parameter of a Rayleigh distribution, such as the maximum likelihood method, method of moments, and local frequency ratio method of estimation [3], [12]. The latter was used since the image histogram provides the frequencies required by the method [12]. The K-distributed envelope PDF is defined by

$$
f(h)=\frac{b(b h)^{v} K_{v-1}(b h)}{2^{v-1} \Gamma(v)} u(h)
$$

where $v$ is the shape parameter of the distribution, $b$ denotes the scale parameter of the distribution, $K_{N}$ is the $N$ th-order modified Bessel function of the second kind and $u(h)$ is the unit step function. $\Gamma(\cdot)$ is the Gamma function that generalizes the factorial function to nonintegral, negative, and complex arguments [13]. The shape and scale parameters were calculated by using higher order and fractional moments [14].

\section{MATHEMATICAL FORMULATION}

A similar nomenclature as that used in [5] is adopted here to formulate the expressions for the implemented likelihood ratio test. Let the subscripts $x$ and $y$ stand for reference and surveillance SAR images, respectively. Hence, the corresponding pair of target, clutter, and noise signals could be denoted by the complex vectors

$$
\vec{s}=\left[\begin{array}{l}
s_{x} \\
s_{y}
\end{array}\right] \quad \vec{c}=\left[\begin{array}{l}
c_{x} \\
c_{y}
\end{array}\right] \quad \vec{n}=\left[\begin{array}{l}
n_{x} \\
n_{y}
\end{array}\right] .
$$

Using (3), two hypotheses are taken into account: the null hypothesis $\left(H_{0}\right)$, which considers no changes in the two SAR images being analyzed, and the alternative hypothesis $\left(H_{1}\right)$, which considers the occurrence of changes in the SAR images. These changes can be related to the existence of targets in a SAR image. $H_{0}$ and $H_{1}$ are expressed as follows:

$$
\begin{aligned}
& H_{0}: \vec{z}_{0}=\left[\begin{array}{l}
x_{0} \\
y_{0}
\end{array}\right]=\left[\begin{array}{l}
c_{x}+n_{x} \\
c_{y}+n_{y}
\end{array}\right] \\
& H_{1}: \vec{z}_{1}=\left[\begin{array}{l}
x_{1} \\
y_{1}
\end{array}\right]=\left[\begin{array}{l}
s_{x}+c_{x}+n_{x} \\
s_{y}+c_{y}+n_{y}
\end{array}\right]
\end{aligned}
$$

where $\vec{z}_{0}$ and $\vec{z}_{1}$ are the complex vectors. The subscript " 0 " indicates the null hypothesis, and the subscript " 1 " indicates the alternative hypothesis. Each of the vectors $\vec{z}_{0}$ and $\vec{z}_{1}$ contains a reference and a surveillance SAR image denoted by $x$ and $y$, respectively. A statistical model for the two hypotheses is implemented by using a likelihood ratio test [11], [13] according to the Neyman-Pearson criterion

$$
\Lambda(z)=\frac{P\left(\vec{z}_{1} \mid H_{1}\right)}{P\left(\vec{z}_{0} \mid H_{0}\right)} \underset{H_{1}}{\stackrel{H_{0}}{\gtrless}} \lambda
$$

where $P$ is the conditional probability under hypothesis $H_{0}$ or $H_{1}$, and $\lambda$ is the threshold value determined according to a required probability of false alarm. $\Lambda(z)$ is the likelihood ratio test that is applied considering incoherent change detection since only amplitude SAR image data are included in the data set. Thus, the phase of the complex numbers in (4) is not available.

In this letter, a probability distribution of targets in SAR images is not assumed. The statistics related to only the background of the image [3], [11] are taken into account for 
the conditional probability under $H_{1}$ in (5). Thus, hypothesis $H_{1}$ should be verified by subtracting the target signal $\vec{s}$ from the SAR images. To compute the test statistic, the target signal $s_{x}$ is assumed to be zero. The reverse triangle inequality for complex numbers, $\left|y_{1}-s_{y}\right| \geq\left|y_{1}\right|-\left|s_{y}\right|$, is observed to obtain an estimate for $s_{y}$, denoted as $\tilde{s_{y}}$

$$
\left|y_{1}\right|-\left|\tilde{s_{y}}\right|=\left|y_{1}-s_{y}\right| \text {. }
$$

Since $\left|y_{1}-s_{y}\right|$ is a positive value, $0 \leq\left|\tilde{s_{y}}\right| \leq\left|y_{1}\right|$.

\section{Bivariate Rayleigh Distribution}

Since the new likelihood ratio test is dedicated to amplitudeonly data [3], the conditional probability under $H_{0}$ is formulated by using the Rayleigh distribution [15] as follows:

$$
\begin{aligned}
P\left(\left|\vec{z}_{0}\right| \mid H_{0}\right)= & \frac{4\left|x_{0}\right|\left|y_{0}\right|}{\Omega_{x} \Omega_{y}\left(1-\rho^{2}\right)} \\
& \times \exp \left\{-\frac{\left(\left|x_{0}\right| / \sqrt{\Omega_{x}}\right)^{2}+\left(\left|y_{0}\right| / \sqrt{\Omega_{y}}\right)^{2}}{\left(1-\rho^{2}\right)}\right\} \\
& \times I_{0}\left(\frac{\rho}{1-\rho^{2}} \frac{2\left|x_{0}\right|\left|y_{0}\right|}{\sqrt{\Omega_{x} \Omega_{y}}}\right) .
\end{aligned}
$$

Since the target distribution is not known, the statistics related to only the background of the image [3], [11] are considered to obtain the expression for the conditional probability under $H_{1}$

$$
\begin{aligned}
& P\left(\left|\vec{z}_{1}\right| \mid H_{1}\right) \\
& =\frac{4\left|x_{1}-s_{x}\right|\left|y_{1}-s_{y}\right|}{\Omega_{x} \Omega_{y}\left(1-\rho^{2}\right)} \\
& \quad \times \exp \left\{-\frac{\left(\left|x_{1}-s_{x}\right| / \sqrt{\Omega_{x}}\right)^{2}+\left(\left|y_{1}-s_{y}\right| / \sqrt{\Omega_{y}}\right)^{2}}{\left(1-\rho^{2}\right)}\right\} \\
& \quad \times I_{0}\left(\frac{\rho}{1-\rho^{2}} \frac{2\left|x_{1}-s_{x}\right|\left|y_{1}-s_{y}\right|}{\sqrt{\Omega_{x} \Omega_{y}}}\right) .
\end{aligned}
$$

In (7) and (8), $\rho$ is the power correlation coefficient, $\Omega_{x}=$ $\overline{|x|^{2}}, \Omega_{y}=\overline{|y|^{2}}$, and $x$ and $y$ are the reference and surveillance images, respectively. $I_{0}$ is the modified Bessel function of the first order. The signals $s_{x}$ and $s_{y}$ are related to target backscattering and are estimated a priori by using known target amplitudes. $s_{x}$ is assumed to be zero since the reference image is considered to have no targets. The estimate for target signal $s_{y}$, i.e., $\tilde{s_{y}}$, is the effective target amplitude used to evaluate the PDF performance. To evaluate the performance of the implemented approaches, the data provided by CARABAS, i.e., experimental data collected during the measurement campaigns in Sweden [2], are used in this letter. Thus, under the assumption of a bivariate Rayleigh PDF, the test according to (5) can be expressed by

$$
\begin{aligned}
\Lambda(\vec{z})=\frac{\left|y_{1}-s_{y}\right|}{\left|y_{0}\right|} \times \exp \left\{\frac{\left(\frac{\left|y_{0}\right|}{\sqrt{\Omega_{y}}}\right)^{2}}{1-\rho^{2}}-\frac{\left(\frac{\left|y_{1}-s_{y}\right|}{\sqrt{\Omega_{y}}}\right)^{2}}{1-\rho^{2}}\right\} \\
\times \frac{I_{0}\left(\frac{\rho}{1-\rho^{2}} \frac{2\left|x_{1}\right|\left|y_{1}-s_{y}\right|}{\sqrt{\Omega_{x} \Omega_{y}}}\right)}{I_{0}\left(\frac{\rho}{1-\rho^{2}} \frac{2\left|x_{0}\right|\left|y_{0}\right|}{\sqrt{\Omega_{x} \Omega_{y}}}\right)} .
\end{aligned}
$$

\section{K-DistRIBUTION FROM SIRP}

When the radar range resolution is increased, the background clutter may no longer be modeled accurately as a Gaussian random variable. If the clutter is non-Gaussian and correlated, many different joint PDFs of the clutter samples can result in the same set of marginal distributions having the specified non-Gaussian character. The specification of the multivariate PDF is generally a nontrivial problem with no simple best solution [16]. The theory of SIRPs provides a mechanism to obtain the joint PDF of the correlated, non-Gaussian clutter samples. [16] and [17] show that the corresponding general expression for the $N$ th-order PDF of an SIRP, whose envelope is $\mathrm{K}$-distributed, is obtained by

$$
\begin{aligned}
f_{N}(x)=|C|^{-\frac{1}{2}}(\pi)^{-\frac{N}{2}} \Gamma^{-1} & (v) v^{-\frac{N}{2}} 2^{(1-v)} \\
& \times(\sqrt{2 v q})^{\nu-\frac{N}{2}} K_{\nu-\frac{N}{2}}(\sqrt{2 v q})
\end{aligned}
$$

where $\mu$ and $C$ are the mean and covariance matrix of a complex or real vector $\vec{x}=\left[x_{1}, x_{2}, \ldots, x_{N}\right]^{T}, N>1$, called a spherically invariant random variable, whose characteristics are shown in [17]. Taking into account the null hypothesis, the following expression represents the conditional probability under $H_{0}$ when using the $\mathrm{K}$-distribution according to (10):

$$
\begin{aligned}
P\left(\left|\vec{z}_{0}\right| \mid H_{0}\right)=|C|^{-\frac{1}{2}}(\pi)^{-\frac{N}{2}} \Gamma^{-1}(v) v^{-\frac{N}{2}} 2^{(1-v)} & \\
& \times\left(\sqrt{2 v q_{0}}\right)^{\nu-\frac{N}{2}} K_{\nu-\frac{N}{2}}\left(\sqrt{2 v q_{0}}\right)
\end{aligned}
$$

where $q_{0}=\left(\left|\vec{z}_{0}\right|-\vec{\mu}\right)^{T} C^{-1}\left(\left|\vec{z}_{0}\right|-\vec{\mu}\right), \vec{\mu}$ is the mean vector, and $C$ is the covariance matrix. $v$ is the shape parameter, which is estimated from the reference image used to implement the test presented by (5). The method used to estimate $v$ is the higher order and fractional moments [14]. The corresponding equation for conditional probability under $H_{1}$ is expressed by

$$
\begin{aligned}
P\left(\left|\vec{z}_{1}\right| \mid H_{1}\right)=|C|^{-\frac{1}{2}}(\pi)^{-\frac{N}{2}} \Gamma^{-1}(v) v^{-\frac{N}{2}} 2^{(1-v)} & \\
& \times\left(\sqrt{2 v q_{1}}\right)^{\nu-\frac{N}{2}} K_{\nu-\frac{N}{2}}\left(\sqrt{2 v q_{1}}\right)
\end{aligned}
$$

where $q_{1}=\left[\left(\left|\vec{z}_{1}\right|-\vec{s}\right)-\vec{\mu}\right]^{T} C^{-1}\left[\left(\left|\vec{z}_{1}\right|-\vec{s}\right)-\vec{\mu}\right]$. Thus, under the assumption of a bivariate K-distributed envelope PDF, the test according to (5) can be expressed by

$$
\Lambda(\vec{z})=\frac{\left(q_{1}\right)^{\frac{v}{2}-N} K_{v-\frac{N}{2}}\left(\sqrt{2 v q_{1}}\right)}{\left(q_{0}\right)^{\frac{v}{2}-N} K_{v-\frac{N}{2}}\left(\sqrt{2 v q_{0}}\right)} .
$$

\section{Vi. Comparison Tests}

The likelihood ratio test using the bivariate Rayleigh distribution is expressed by (9), and (13) expresses the test when using the K-distribution. Both equations are implemented by observing the condition in (6) since amplitude-only data are available. These equations are applied to subimages resulting from segmenting the SAR image into blocks of $100 \times 100$ pixels. Therefore, $\rho, \Omega_{x}$, and $\Omega_{y}$ from (9) and $C$ and $\vec{\mu}$ from (13) characterize a specific region of the SAR image since they are estimated using data from a specific block. The target signal $\tilde{s_{y}}$ is evaluated for values ranging from 0.3 to 2.25 in steps of 0.01 . The threshold $\lambda$ from (5) is observed for values ranging from 1 to 100 in steps of 10 and from $10^{2}$ to $10^{25}$ with exponent steps of 1 . 
TABLE I

FALSE Alarm COMPARISON BASED ON THE DETECTION RESULTS FOR $\tilde{s_{y}}=1.25$

\begin{tabular}{|c|c|c|c|c|c|c|c|c|c|}
\hline \multicolumn{2}{|c|}{$\begin{array}{c}\begin{array}{c}\text { Surveillance } \\
\text { Image }\end{array} \\
\end{array}$} & \multicolumn{2}{|c|}{$\begin{array}{l}\text { Reference } \\
\text { Image }\end{array}$} & \multirow{2}{*}{$\begin{array}{l}\text { Known } \\
\text { Targets }\end{array}$} & \multirow{2}{*}{$\begin{array}{l}\text { Area } \\
\mathrm{km}^{2}\end{array}$} & \multicolumn{2}{|c|}{$\begin{array}{c}\text { Rayleigh } \\
\text { Distribution }\end{array}$} & \multicolumn{2}{|c|}{$\begin{array}{c}\mathbf{K} \\
\text { Distribution }\end{array}$} \\
\hline Mission & Pass & Mission & Pass & & & $n_{d}$ & $n_{f a}$ & $n_{d}$ & $n_{f a}$ \\
\hline 2 & 1 & 3 & 1 & 25 & 6 & 24 & 65 & 25 & 1 \\
\hline 3 & 1 & 4 & 1 & 25 & 6 & 18 & 135 & 21 & 3 \\
\hline 4 & 1 & 5 & 1 & 25 & 6 & 25 & 80 & 25 & 0 \\
\hline 5 & 1 & 2 & 1 & 25 & 6 & 25 & 128 & 25 & 3 \\
\hline 2 & 2 & 4 & 2 & 25 & 6 & 25 & 192 & 25 & 0 \\
\hline 3 & 2 & 5 & 2 & 25 & 6 & 25 & 227 & 25 & 2 \\
\hline 4 & 2 & 2 & 2 & 25 & 6 & 25 & 270 & 25 & 1 \\
\hline 5 & 2 & 3 & 2 & 25 & 6 & 24 & 236 & 22 & 2 \\
\hline 2 & 3 & 5 & 3 & 25 & 6 & 25 & 96 & 25 & 2 \\
\hline 3 & 3 & 2 & 3 & 25 & 6 & 20 & 156 & 22 & 0 \\
\hline 4 & 3 & 3 & 3 & 25 & 6 & 25 & 58 & 25 & 2 \\
\hline 5 & 3 & 4 & 3 & 25 & 6 & 24 & 75 & 25 & 1 \\
\hline 2 & 4 & 3 & 4 & 25 & 6 & 25 & 217 & 24 & 1 \\
\hline 3 & 4 & 4 & 4 & 25 & 6 & 25 & 256 & 25 & 0 \\
\hline 4 & 4 & 5 & 4 & 25 & 6 & 25 & 234 & 22 & 0 \\
\hline 5 & 4 & 2 & 4 & 25 & 6 & 24 & 278 & 21 & 0 \\
\hline 2 & 5 & 4 & 5 & 25 & 6 & 25 & 58 & 25 & 1 \\
\hline 3 & 5 & 5 & 5 & 25 & 6 & 15 & 50 & 16 & 9 \\
\hline 4 & 5 & 2 & 5 & 25 & 6 & 25 & 123 & 25 & 1 \\
\hline 5 & 5 & 3 & 5 & 25 & 6 & 25 & 218 & 25 & 69 \\
\hline 2 & 6 & 5 & 6 & 25 & 6 & 25 & 102 & 25 & 1 \\
\hline 3 & 6 & 2 & 6 & 25 & 6 & 21 & 119 & 22 & 0 \\
\hline 4 & 6 & 3 & 6 & 25 & 6 & 25 & 78 & 25 & 1 \\
\hline 5 & 6 & 4 & 6 & 25 & 6 & 23 & 143 & 25 & 0 \\
\hline \multicolumn{4}{|c|}{ Total } & 600 & 144 & 568 & 3594 & 570 & 100 \\
\hline
\end{tabular}

\section{A. Database Description}

The data set consisting of 24 magnitude SAR images is a subset of the data collected during a flight campaign held in northern Sweden in 2002 [2], [5]. Each image corresponds to a rectangle of the same area of $3 \mathrm{~km} \times 2 \mathrm{~km}$. The illumination of the radar was done using three different flight tracks given three image stacks containing eight images in each stack. The targets are 25 terrain vehicles, which were deployed at the upper left or lower right of the ground scene and concealed by foliage. In Fig. 1, as an example, we indicated the target positions with a rectangle. The images were taken by CARABAS II, the second-generation UWB SAR mounted on a Sabreliner airplane. The system was operated in the frequency range of $20-90 \mathrm{MHz}$, with corresponding wavelengths between 3.3 and $15 \mathrm{~m}$. The resolution of the radar is approximately $2.5 \times 2.5 \mathrm{~m}$, and each pixel in the image corresponds to an area of $1 \mathrm{~m} \times 1 \mathrm{~m}$ on the ground. Therefore, the resolution is smaller than the wavelength. More details can be found in [2]. The images are used as surveillance or reference images, as presented in Table I.

\section{B. Test Performance}

We do not use a multilooking approach nor filter the data set images to decrease the number of false alarms. Therefore, we can have a better tradeoff between resolution and detection performance. It also gives a more fair comparison between the distributions. Hence, after applying the likelihood ratio test, the resulting image is binarized according to the threshold $\lambda$. Receiver operating characteristic (ROC) curves are used to verify the algorithm performance. The probability of detection $\left(P_{d}\right)$ and FAR are calculated as explained in [5].

Fig. 3 presents the results for tests based on the Rayleigh distribution. The best result is obtained for effective target $\tilde{s_{y}}$ equal to 1.25 . The probability of detection decreases for $\tilde{s_{y}} \neq 1.25$. Thus, regarding the values attributed to $\tilde{s_{y}}$ in Fig. 3, $\tilde{s_{y}}=1.25$ represents the best approximation to the real

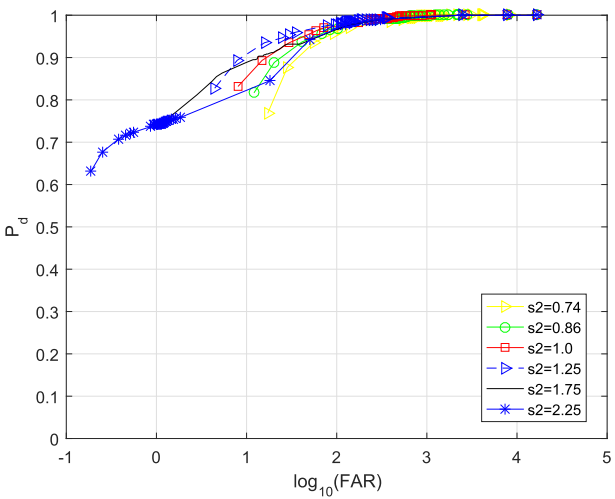

Fig. 3. Select ROC curves obtained by the method based on the Rayleigh distribution.

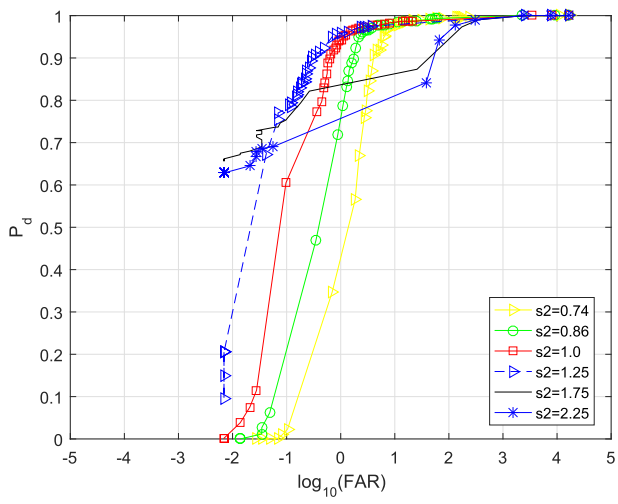

Fig. 4. Select ROC curves obtained by the method based on the K-distribution.

value of the target amplitude, as a consequence, the best probability of detection is obtained. Target detection (5) is also implemented using the test derived for the K-distribution (13). The test is done in the similar way to that of the Rayleigh distribution by using an effective target amplitude $\tilde{s_{y}}=1.25$. The results are presented in Fig. 4. Again, the probability of detection decreases for observed values of $\tilde{s_{y}} \neq 1.25$.

\section{Comparison of the Rayleigh and K-Distributions}

Table I compares the results of the implementation of (5) employing the Rayleigh and K-distributions. The comparison takes into account an equal number of total detections for the two distributions. $\tilde{s_{y}}=1.25$ was selected since that value produced the best results for the total number of detections, i.e., 568 for the Rayleigh distribution and 570 for the K-distribution, when applying thresholds of $10^{12}$ and 20, respectively. Thus, the total number of false alarms for test statistic based on the Rayleigh distribution was 3594. For the K-distribution, a total of 100 false alarms occurred. The K-distribution presents the best results for the probability of detection versus FAR. Figs. 3 and 4 show that for a rate of one false alarm per $\mathrm{km}^{2}$, the corresponding probability of detection is approximately $96 \%$. For the same probability of detection, the Rayleigh distribution presents a rate of approximately 32 false alarms per $\mathrm{km}^{2}$. Table I, where $n_{d}$ is the number of detections and $n_{f a}$ is the number of false alarms, shows the highest number of false alarms resulting from the test of the 
surveillance image for mission 5 and pass 5 and the reference image for mission 3 and pass 5 when using the K-distribution. Both images are responsible for $69 \%$ of the false alarms, which could be a consequence of the way the images were collected. According to [2], both images were collected with a flight heading of $230^{\circ}$, which positioned the antenna main lobe toward a TV transmitter located next to the test area. Hence, high radio frequency interference was received, deteriorating the quality of the referred pair of images.

\section{CONCLUSION}

In this letter, two approaches to a likelihood ratio test for incoherent change detection in SAR images are analyzed. The first approach employs a circular bivariate Rayleigh distribution, and the second uses a K-distribution based on the theory of SIRPs. The results indicate that the K-distribution presents the best probability of detection and the lowest FAR, which agrees with Fig. 2, where the pixel distribution of the SAR image is better characterized by the K-distribution PDF envelope. In addition, Fig. 3 indicates that the likelihood ratio test using the Rayleigh distribution presents small variations in detection probability for different values of $\tilde{s_{y}}$, which characterizes the robustness of the methodology. Table I shows that when considering an approximately equal number of total detections for the two distributions, the total number of false alarms is much smaller for the K-distribution than it is for the Rayleigh distribution, indicating that the K-distribution is more appropriate for the pixel distribution of SAR images. Table I presents the results for an effective target amplitude $\tilde{s_{y}}=1.25$. This value of $\tilde{s_{y}}$ was chosen because it produced the best values for the total number of detections, i.e., 568 for the Rayleigh distribution and 570 for the K-distribution, when applying thresholds of $10^{12}$ and 20 , respectively.

\section{REFERENCES}

[1] Y. Huang, L. Ferro-Famil, and A. Reigber, "Under-foliage target detection using multi-baseline 1-band polinSAR data," in Proc. IGARSS, Jul. 2013, pp. 2353-2356.
[2] M. Lundberg, L. M. H. Ulander, W. E. Pierson, and A. Gustavsson, "A challenge problem for detection of targets in foliage," Proc. SPIE, vol. 6237, p. 62370K, May 2006.

[3] C. Oliver, Understanding Synthetic Aperture Radar Images. Norwood, MA, USA: Artech House, 1998.

[4] R. J. Radke, S. Andra, O. Al-Kofahi, and B. Roysam, "Image change detection algorithms: A systematic survey," IEEE Trans. Image Process., vol. 14, no. 3, pp. 294-307, Mar. 2005.

[5] L. M. H. Ulander, M. Lundberg, W. Pierson, and A. Gustavsson, "Change detection for low-frequency SAR ground surveillance," IEE Proc.-Radar, Sonar Navigat., vol. 152, no. 6, pp. 413-420, Dec. 2005.

[6] M. Gong, Z. Zhou, and J. Ma, "Change detection in synthetic aperture radar images based on image fusion and fuzzy clustering," IEEE Trans. Image Process., vol. 21, no. 4, pp. 2141-2151, Apr. 2012.

[7] R. Vijayalakshm and K. M. Kumar, "Analysis of SAR images with various change detection techniques," Int. J. Emerg. Technol. Eng., vol. 1, no. 2, pp. 52-58, Mar. 2014.

[8] H. Aghababaeea, J. Amini, and Y. C. Tzengb, "Improving change detection methods of SAR images using fractals," Scientia Iranica, vol. 20, no. 1, pp. 15-22, Feb. 2013.

[9] O. A. Ajadi, F. J. Meyer, and P. W. Webley, "Change detection in synthetic aperture radar images using a multiscale-driven approach," Remote Sens., vol. 8, no. 6, p. 482, Jun. 2016.

[10] B. Cui, Y. Y. L. Zhang, and X. Cai, "A SAR intensity images change detection method based on fusion difference detector and statistical properties," in Proc. ISPRS Ann. Photogram., Remote Sens. Spatial Inf. Sci., Wuhan, China, Sep. 2017, pp. 439-443.

[11] W.-S. Chen and I. S. Reed, "A new CFAR detection test for radar," Digit. Signal Process., vol. 1, no. 4, pp. 198-214, Oct. 1991.

[12] A. Moya, J. C. Suárez, P. J. Amado, S. Martin-Ruíz, and R. Garrido, "Frequency ratio method for seismic modeling of $\gamma$ doradus stars," Astron. Astrophys., vol. 432, no. 1, pp. 189-198, 2005.

[13] A. Papoulis and S. U. Pillai, Probability, Random Variables and Stochastic Processes (Electrical Engineering. Communications and Signal Processing), vol. 576, 2nd ed. New York, NY, USA: McGraw-Hill, 1984.

[14] D. R. Iskander and A. M. Zoubir, "Estimation of the parameters of the K-distribution using higher order and fractional moments [radar clutter]," IEEE Trans. Aerosp. Electron. Syst., vol. 35, no. 4, pp. 1453-1457, Oct. 1999.

[15] M. K. Simon and M.-S. Alouini, Digital Communication Over Fading Channels. Hoboken, NJ, USA: Wiley, 2005.

[16] M. Rangaswamy, "Spherically invariant random processes for radar clutter modeling, simulation and distribution identification," Ph.D. dissertation, Dept. Elect. Comput. Eng., Syracuse Univ., Syracuse, NY, USA, Dec. 1992.

[17] E. Conte and M. Longo, "Characterisation of radar clutter as a spherically invariant random process," IEE Proc. F-Commun., Radar Signal Process., vol. 134, no. 2, pp. 191-197, Apr. 1987. 